\title{
Factores claves en la gestión de mercadeo que inciden en la competitividad del sector de comunicaciones gráficas de la ciudad de Barranquilla,Colombia
}

Key factors in marketing

management which affect the

competitiveness of the graphic

communication sector in

Barranquilla city, Lolombia

Facteurs clés de la gestion marketing influant sur la compétitivité du secteur de la communication graphique de la ville de Barranquilla, Colombie

Factores chaves na gestão de marketing, que incidem na competitividade do setor de comunicações gráficas na cidade de Barranquilla, Colômbia
* Doctorado en Ciencias Sociales, Universidad del Zulia; Magister en Mercadeo, Universidad Autónoma del Caribe; Especialista en Mercadeo, Universidad Autónoma del Caribe, Barranquilla; Ingeniero Industrial, Universidad Autónoma del Caribe, Barranquilla 


\section{RESUMEN}

En el presente artículo, se identifican los factores claves de la gestión de mercadeo que inciden en la competitividad de las empresas del sector gráfico ubicadas en Barranquilla, según el criterio de los empresarios. Mediante un estudio exploratorio, descriptivo, se aplicó un instrumento a un grupo de empresarios del sector, obteniéndose como resultado que seis factores del área son los que inciden en la competitividad. Finalmente, se realizó un análisis correlacional para establecer dependencias entre los factores e identificar la influencia de la teoría administrativa sobre mercadeo y la dependencia entre los factores a la hora de diseñar estrategias de mercadeo.

\section{ABSTRACT}

This article describes the key factors of marketing management that impact the competitiveness of the graphic companies located in Barranquilla, based on managers' opinions. Following a method of exploration and description analysis, an instrument was applied to a group of managers belonging to this economic sector, showing that there are six factors which affect competitiveness. Finally, a correlational analysis was performed to establish the relationships among all these factors and also to describe the influence of the managerial theory on marketing and the relationships among all these factors when designing marketing strategies.

\section{RESUMÉÉ}

Cet article identifie les facteurs clés de la gestion marketing qui influent, selon les chefs d'entreprise, sur la compétitivité des entreprises du secteur graphique de Barranquilla. Lors de cette étude expérimentale et descriptive, un outil d'analyse a été soumis à un groupe d'entrepreneurs du secteur permettant de définir que 6 facteurs de la région ont un impact sur la compétitivité. Une analyse de corrélation a également été réalisée afin d'établir des interdépendances entre les facteurs pour identifier l'influence de la théorie administrative au moment de la conception de stratégies marketing.

\section{RESUMO}

Neste artigo identificam-se os factores chaves da gestão de marketing que incidem na competitividade das empresas do setor gráfico localizadas em Barranquilla, segundo o critério dos empresários. Através de um estudo exploratório - descritivo, aplicou-se um instrumento em um grupo de empresários do setor, obtendo como resultado que 6 fatores da área são os que incidem na competitividade. Finalmente realizou-se uma análise correlacional para estabelecer as dependências entre os factores e identificar a influência da teoria administrativa sobre marketing $e$ a dependência entre os factores no momento de desenhar estratégias de marketing

\section{Palabras claves}

Competitividad

Gerencia de mercadeo Comunicaciones gráficas Innovación empresarial

\section{Key words}

Competitiveness

Marketing management Graphic communications Company innovation

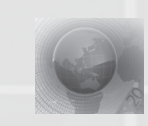

\author{
Compétitivité \\ Gestion marketing \\ Communications graphiques \\ Innovation entrepreneuriale
}




\section{INTRODucción}

L a ciudad de Barranquilla, cuenta con la presencia de un grupo de empresas pertenecientes al sector de la comunicación gráfica, incluido entre los de categoría mundial en Colombia y del cual se requiere caracterizar y establecer los grados de competitividad para concluir si se encuentra al mismo nivel respecto del resto del sector en el país, el cual pasa por un excelente momento en Latinoamérica. Mercadeo, es el área que debe responder por la competitividad de una empresa, por la esencia misma del significado de competitividad y por ende, su gestión ejerce un efecto para que la empresa pueda vencer o perder ante los competidores.

Colombia, a través de normativas como la política CONPES 3527, promueve el desarrollo de sectores más competitivos, que estén al nivel de los líderes o referentes internacionales, a los cuales el Estado los cataloga como sectores de clase mundial. La industria de la comunicación gráfica, se considera de clase mundial en Colombia, gracias a los buenos resultados obtenidos por empresas del sector ubicadas en el territorio nacional, el posicionamiento a nivel latinoamericano y el buen nombre construido por empresas líderes, como es el caso de las pertenecientes al grupo Carvajal. Este sector, juega un papel valioso para otros sectores estratégicos, así como también lo juega el sector de servicios logísticos, puesto que ellos son los aliados en la estrategia de comunicación de toda organización. Así como el sector de servicios logísticos hace posible que los productos estén a tiempo donde el consumidor lo desea y el sector financiero facilita los recursos económicos para la operación, el sector de las comunicaciones gráficas, hace posible que la imagen de las empresas y de sus productos, pueda ser visualizada por los clientes, incidiendo en la competitividad de las empresas.

Por la naturaleza misma de las comunicaciones gráficas, está presente en gran parte del territorio colombiano. El departamento delAtlántico, no es la excepción. Sin embargo, siendo este uno de los departamentos representativos de la economía colombiana, el sector de las comunicaciones gráficas no es una de las especialidades ni de los referentes en la economía local, a pesar de la presencia de grandes compañías del sector a nivel nacional en la región.

Es así como el sector de las comunicaciones gráficas es uno de los más representativos de Colombia, surge la iniciativa de elaborar un documento de investigación que analice su competitividad en el departamento del Atlántico desde la gestión de mercadeo, estableciendo cuáles son esos factores claves de su gestión, que se llevan o no a cabo por las empresas del sector en el departamento, que afectan la competitividad proyecto que a la vez, se justifica por la poca literatura local sobre temas relacionados, en especial en cuanto al aporte del mercadeo a la competitividad empresarial. 


\section{REVISIÓN LITERARIA}

$\mathrm{E}$ ntre los factores que determinan la supervivencia de las empresas, se encuentran la capacidad de tomar decisiones acertadas con base en la gerencia estratégica, la creación de valor para el cliente y en especial,

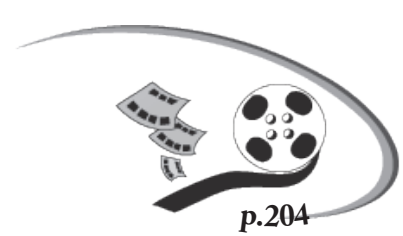
por su productividad y competitividad. Las teorías sobre la gerencia estratégica de mercadeo, están ligadas a estos tres factores porque contribuyen en el proceso de planeación para la creación de valor de manera eficiente y orienta las acciones de la organización hacia su mercado objetivo (Metzger y Donaire, 2007), lo cual permite tomar decisiones acertadas que contribuyen a la supervivencia en los mercados.

Se entiende por gestión estratégica de mercadeo o gestión de mercadeo, como aquella parte de la planeación estratégica donde se utilizan las teorías y técnicas del mercadeo para la elaboración de la estrategia corporativa, con la cual las organizaciones alcanzan los objetivos finales y con estos, se determina el éxito del negocio. Según la Asociación Americana de Mercadeo (AMA, por sus siglas en ingles), la gerencia de mercadeo se define como: "el proceso de establecimiento de objetivos de Marketing de una organización (teniendo en cuenta los recursos internos y las oportunidades de mercado), la planificación y ejecución de actividades para cumplir con estas metas, y medir el progreso hacia su consecución. Comentario: el proceso es continuo y repetitivo (como dentro de un ciclo de planificación) para que la organización de forma continua pueda adaptarse a los cambios internos y externos que crean nuevos problemas y oportunidades".

La gestión de mercadeo, aunque en esencia sea muy parecida en todos los escenarios globales, debe tener en cuenta factores que son propios de cada región, o de cada grupo de consumidor, para así adaptarse a esa situación y establecer la gestión de mercadeo adecuada. En la literatura local, se encuentran pocos estudios enfocados a resaltar la efectividad de la gestión de mercadeo en el crecimiento empresarial. Zapata (2001) en su investigación "La efectividad del mercadeo en las pequeñas y medianas empresas (PyMEs) de los sectores industriales y de servicios de Boyacá, Colombia",

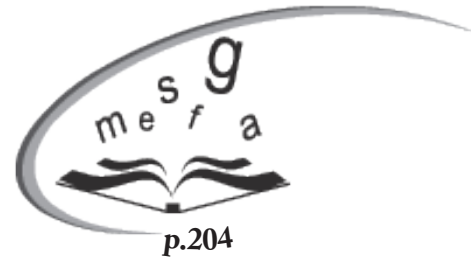
donde se establece que las de la región para la época, manejaban buenos índices de ventas, aunque no realizaban gestión de mercadeo. Gómez (2010) en su investigación "La productividad y competitividad de la industria de confecciones infantiles de Bucaramanga, en la dinámica de la gestión estratégica", destaca la importancia de la construcción de marca y la definición de líneas de producto con las cuales competir en el mercado para una muestra de 61 empresas de la región estudiada. Por otra parte, Barrios y López (2007) en su investigación "Diseño de un modelo base de estructura organizacional para PyMEs exportadoras del sector de confecciones de Barranquilla, con enfoque asociativo", propone un modelo base que contempla la existencia de un departamento de mercadeo y ventas agrupado al área comercial, junto a comercio exterior y logística. Un documento investigativo que aporta interesante información al proyecto, es el de Matiz et. al (2011) titulado: "Turbulencia empresarial en Colombia: el caso del sector hotelero", el cual concluye que la estrategia de creación de nuevos nichos es la recomendada para el sector y generaría una vida financiera estable, así como la constante innovación en producto, dada la alta turbulencia en el sector por los cambios del entorno, la variación es a nivel macro y la fuerte rivalidad en precios y promociones. 


\section{METODOLOGÍA}

$\mathbf{L}$ a metodología aplicada es exploratoria descriptiva. El nivel exploratorio se justifica debido a la escasez de literatura local sobre el tema, lo que permite concluir que el problema no ha sido abordado anteriormente o por lo menos, no se conoce un trabajo formal al respecto. Abarca también el nivel descriptivo, en la medida que se describe la labor de mercadeo realizada en la población de estudio y se identifican los factores claves que distinguen al tipo de gestión de mercadeo realizada por las empresas del sector.
En cuanto a las actividades, se parte de una revisión de informes técnicos y académicos relacionados con las tendencias del mercado que hacen competitivas a las empresas en el sector a nivel internacional y en Colombia. Con las conclusiones sobre las tendencias, se diseñó una entrevista para aplicar a expertos en el área de mercadeo, que permitiera identificar los factores claves de la gestión de mercadeo que ayudan a una empresa de cualquier sector, a ser competitiva. El resultado obtenido, permitió construir una escala de medición que fue sometida ante un grupo de 25 representantes de empresas tipo PyME, que conforman el sector en la ciudad de Barranquilla,

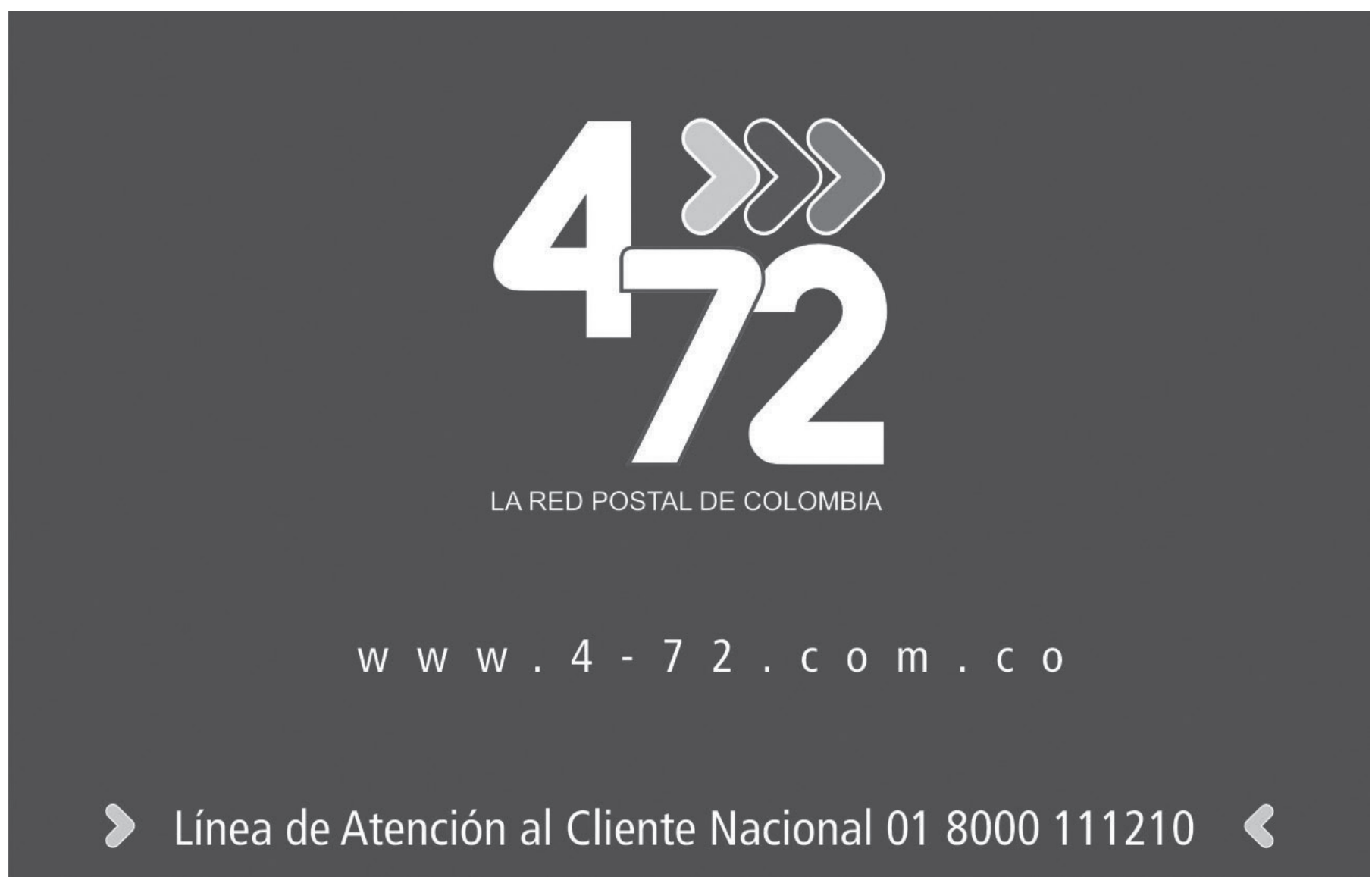




\section{RESULTADOS}

\subsection{Principales tendencias de mercado obtenidas a través de revisión}

Mediante la revisión de informes colombianos e internacionales, se obtuvieron las conclusiones, sobre cuáles son

las principales tendencias de mercado para el sector de las comunicaciones gráficas (tabla 1).

Tabla 1. Resumen diagnóstico de tendencias de mercado para el sector de comunicaciones gráficas

\begin{tabular}{|c|c|}
\hline Variable de mercadeo & Datos relevantes del sector gráfico en Colombia y a nivel internacional \\
\hline \multirow{4}{*}{ Tendencias de consumo } & $\begin{array}{l}\text { - Interés por el consumo de contenidos } \\
\text { digitales. }\end{array}$ \\
\hline & - La demanda del transpromo. \\
\hline & - La conciencia ambiental. \\
\hline & - La impresión por demanda. \\
\hline Estrategias de producto & $\begin{array}{l}\text { - Oferta de nuevos líneas de producto, como las aplicaciones de seguridad y los libros digitales. } \\
\text { - Los servicios de valor agregado, con enfoque en servicios logísticos, es una de las estrategias } \\
\text { llevadas a cabo en empresas de alta competitividad internacional. }\end{array}$ \\
\hline Canales de marketing & Leve migración hacia la Web como canal, mantiene la fortaleza el canal directo. \\
\hline Canales de comunicación & $\begin{array}{l}\text { Las ferias siguen siendo uno de los principales canales para la divulgación y presentación de las } \\
\text { empresas. }\end{array}$ \\
\hline Tecnologías asociadas & $\begin{array}{l}\text { En Colombia, se presentan atrasos tecnológicos con respecto a los grandes de la industria; sin } \\
\text { embargo, el país se ubica como el tercero de Latinoamérica según exportaciones. } \\
\text { Se requiere mayor inversión para migración a contenidos digitales y productos más amigables al } \\
\text { medio ambiente. }\end{array}$ \\
\hline $\begin{array}{l}\text { Otros datos generales sobre la } \\
\text { industria }\end{array}$ & $\begin{array}{l}\text { Para Colombia, la informalidad empresarial le resta competitividad al sector en el país. La } \\
\text { creatividad está, pero no se materializa a través de un direccionamiento estratégico con enfoque } \\
\text { innovador, promoviendo que se creen más oportunidades o preparando las empresas para afrontar } \\
\text { las tendencias de la industria. A nivel global, el escenario es prometedor hacia medios digitales, } \\
\text { perdiendo cada vez más terreno las impresiones tradicionales, pero que a su vez encuentran } \\
\text { crecimiento en la oferta de valor, como lo es el ofrecer un producto ecoamigable o servicios de } \\
\text { valor agregado que le den una experiencia enriquecedora. }\end{array}$ \\
\hline
\end{tabular}

Fuente. Elaboración propia del autor.

Las empresas del sector en mención dentro de la ciudad de Barranquilla, pertenecen en su mayoría al área de impresión. La impresión comercial se refiere a las flexografías, formas continuas, litografías, editoriales, periódicos y revistas. Estas tres últimas, no se encuentran dentro del área de estudio de este proyecto. En menor medida se encuentran empresas de publicidad en actividades como: imagen corporativa, Branding y estrategias de comunicación. Cabe anotar que en varios casos, parte de las empresas ejercen las dos actividades (impresión y publicidad).

Mediante entrevista semiestructurada a un grupo de cinco empresas se deduce que conocen sobre acciones de Marketing y su importancia para ser más competitivas; sin embargo, aceptan que no las aplican con frecuencia debido a no tener una percepción positiva sobre los 
resultados que el Marketing pueda generar en ellas. En ese sentido, aceptan y reconocen que realizar investigación de mercados, lanzamiento constante de productos nuevos y la planeación estratégica de Marketing dentro de esas organizaciones, es débil.

No son entonces, las estrategias de Marketing las fortalezas de estas empresas, así como las actividades de investigación de mercados mediante técnicas tradicionales, confiando

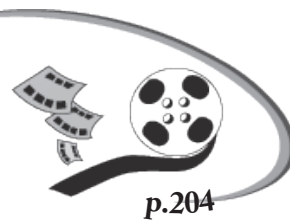
así más en el instinto de negocios que en información objetiva obtenida de indagar en el mercado. No existe el interés por innovar, aunque sí por la diferenciación en los productos a través de servicios de valor agregado pues lo consideran clave para la fidelización de los productos. Esa diferenciación, le da a la creatividad comercial, un nivel de importancia a través de la inyección personalizada de sus productos productos muy ajustados a la medida de cada cliente. Se puede observar, como una estrategia de sobrevivencia en el mercado.

En cuanto al servicio en el proceso de venta, según criterio de los empresarios, la logística manejada genera un buen nivel competitivo en las empresas entrevistadas, manteniéndose en entregas rápidas, calidad en el producto y cumplimiento en lo pactado en cotizaciones a los clientes. Contrario a lo que se cree que puede ser el fuerte en empresas afines al mundo publicitario, las estrategias publicitarias son muy escasas o casi nulas, solo la utilizan al inicio del negocio y consideran que no les es algo rentable porque son más los gastos y poco el efecto en las ventas y utilidades.

\subsection{Factores de la gestión de mercadeo inci- dentes en la competitividad según juicio de los empresarios}

La tabla 2, resume las respuestas de los empresarios en las opciones 4 y 5 , quienes evaluaron de 1 a 5 la incidencia de los factores enlistados en el instrumento de medición, donde: 1 ; no permite ser competitivo, 2; poco competitivo, 3 ; medianamente competitivo, 4 ; es competitivo y 5 ; muy competitivo. Se resaltan en negrilla los resultado que sumados, superan el 50\%.

Se destacan aquellos resultados que superan el 50\% al sumar las respuestas 4 y 5 , lo que indica que la muestra de empresas del sector gráfico en la ciudad de Barranquilla, considera seis factores relacionados con la gestión de mercadeo como los importantes para ser competitivos en el sector.

Después de los anteriores resultados, en la tabla 3, se muestra la trazabilidad existente entre los factores seleccionados por los empresarios y las tendencias de mercado del sector, a nivel Colombia e internacional. 
Tabla 2. Resultados variables por tiempo de funcionamiento

\begin{tabular}{|c|c|c|c|c|c|c|c|}
\hline & & $\begin{array}{l}1 \text { a } 5 \\
\text { años }\end{array}$ & $\begin{array}{c}11 \text { a } 15 \\
\text { años }\end{array}$ & $\begin{array}{c}6 \text { a } 10 \\
\text { años }\end{array}$ & $\begin{array}{l}\text { más de } \\
15 \text { años }\end{array}$ & Subtotal & $\begin{array}{c}\text { Total de la } \\
\text { Muestra }\end{array}$ \\
\hline Factor IM & 4 & $20 \%$ & & $0 \%$ & $0 \%$ & $20 \%$ & $7 \%$ \\
\hline & 5 & $0 \%$ & & $20 \%$ & $60 \%$ & $80 \%$ & $20 \%$ \\
\hline Factor NP & 4 & $14 \%$ & $14 \%$ & $29 \%$ & $29 \%$ & $86 \%$ & $35 \%$ \\
\hline & 5 & $0 \%$ & $0 \%$ & $0 \%$ & $14 \%$ & $14 \%$ & $6 \%$ \\
\hline Factor VA & 4 & $10 \%$ & $10 \%$ & $10 \%$ & $20 \%$ & $50 \%$ & $29 \%$ \\
\hline & 5 & $10 \%$ & $0 \%$ & $20 \%$ & $20 \%$ & $50 \%$ & $29 \%$ \\
\hline Factor DP & 4 & $9 \%$ & & $27 \%$ & $27 \%$ & $64 \%$ & $44 \%$ \\
\hline & 5 & $9 \%$ & & $9 \%$ & $18 \%$ & $36 \%$ & $25 \%$ \\
\hline Factor GL & 4 & $10 \%$ & $10 \%$ & $20 \%$ & $10 \%$ & $50 \%$ & $31 \%$ \\
\hline & 5 & $0 \%$ & $0 \%$ & $10 \%$ & $40 \%$ & $50 \%$ & $31 \%$ \\
\hline Factor CC & 4 & $8 \%$ & $8 \%$ & $0 \%$ & $8 \%$ & $23 \%$ & $19 \%$ \\
\hline & 5 & $8 \%$ & $0 \%$ & $31 \%$ & $38 \%$ & $77 \%$ & $63 \%$ \\
\hline Factor EP & 4 & $0 \%$ & $0 \%$ & $14 \%$ & $0 \%$ & $100 \%$ & $6 \%$ \\
\hline & 5 & & & & & & \\
\hline Factor PM & 4 & $17 \%$ & & $17 \%$ & $17 \%$ & $50 \%$ & $19 \%$ \\
\hline & 5 & $17 \%$ & & $17 \%$ & $17 \%$ & $50 \%$ & $19 \%$ \\
\hline Factor SC & 4 & $20 \%$ & $10 \%$ & $30 \%$ & $20 \%$ & $80 \%$ & $50 \%$ \\
\hline & 5 & $0 \%$ & $0 \%$ & $0 \%$ & $20 \%$ & $20 \%$ & $13 \%$ \\
\hline Factor EM & 4 & & & $20 \%$ & $60 \%$ & $80 \%$ & $25 \%$ \\
\hline & 5 & & & $20 \%$ & $0 \%$ & $20 \%$ & $6 \%$ \\
\hline Factor ARC & 4 & & $11 \%$ & $22 \%$ & $22 \%$ & $56 \%$ & $31 \%$ \\
\hline & 5 & & $0 \%$ & $11 \%$ & $33 \%$ & $44 \%$ & $25 \%$ \\
\hline
\end{tabular}

Fuente. Elaboración propia del autor.

Tabla 3. Trazabilidad factores de mercadeo incidentes en la competitividad con tendencias nacionales e internacionales

\begin{tabular}{|l|c|l|}
\hline \multicolumn{1}{|c|}{$\begin{array}{c}\text { Factor incidente en la } \\
\text { competitividad }\end{array}$} & $\begin{array}{c}\text { \% } \\
\text { obtenido }\end{array}$ & \multicolumn{1}{|c|}{$\begin{array}{c}\text { Trazabilidad con la competitividad a nivel nacional y/o } \\
\text { internacional }\end{array}$} \\
\hline $\begin{array}{l}\text { El aporte de valor agregado en los productos actuales } \\
\text { (VA). }\end{array}$ & 58 & $\begin{array}{l}\text { Tiene relación con las tendencias de mercado del sector tanto } \\
\text { a nivel nacional como internacional. }\end{array}$ \\
\hline Precios agresivos que pueda arriesgar ganancias (DP) & 69 & No tiene relación con las tendencias. \\
\hline $\begin{array}{l}\text { Gestión efectiva en la logística comercial, enfocada a } \\
\text { reducir tiempos de entrega y con precios competitivos } \\
(\mathrm{GL}) .\end{array}$ & 62 & $\begin{array}{l}\text { Tiene relación con las tendencias competitivas de mercado, } \\
\text { más aún, si se miran como valores agregados a ofrecer por } \\
\text { parte de la empresa. }\end{array}$ \\
\hline $\begin{array}{l}\text { La creatividad en la comercialización (CC). } \\
\text { Seguimiento a clientes en pre y pos venta (SC). }\end{array}$ & 62 & $\begin{array}{l}\text { Es una lógica trascendental del negocio, guarda relación pero } \\
\text { internacional, aunque se requiere. }\end{array}$ \\
\hline $\begin{array}{l}\text { Uso de herramientas informáticas para una mejor } \\
\text { administración de los clientes (ARC). }\end{array}$ & 56 & $\begin{array}{l}\text { Las herramientas informáticas tienen una relación, pero con } \\
\text { un perfil más amplio que el de solo administración de clientes. } \\
\text { Se puede conservar. }\end{array}$ \\
\hline
\end{tabular}

Fuente. Elaboración propia del autor. 
Acorde con la tabla anterior, se concluye que los factores VA, GL, CC y ARC, hacen parte de la gestión de mercadeo que presentan mayor incidencia para la competitividad de las empresas, teniendo en cuenta las tendencias del sector y el criterio de los empresarios.

\subsection{Relaciones entre los factores incidentes}

Partiendo de la importancia del pensamiento sistémico en una organización, se procede a hacer un análisis de relación de las distintas variables desde el punto de vista de dos escenarios:
Escenario 1:relacionando todas las variables para encontrar dependencia o independencia entre ellas según el criterio de los empresarios, teniendo como premisa inicial que en una gestión de mercadeo existen relaciones fuertes entre la investigación de mercado para lanzar nuevos productos y/o agregar valor; así mismo, la creatividad en la comercialización empieza a tener una relación directa con las estrategias de mercado a través de Internet o medios móviles. Se resaltan en negritas, las variables con alta dependencia.

Tabla 4. Coeficiente de correlación de Pearson factores de gestión de mercadeo, escenario 1

\begin{tabular}{|l|l|l|l|l|l|l|l|l|l|l|l|}
\hline & \multicolumn{1}{|c|}{ IM } & \multicolumn{1}{|c|}{ NP } & \multicolumn{1}{|c|}{ VA } & \multicolumn{1}{|c|}{ CC } & DP & GL & EP & PM & SC & EM & ARC \\
\hline IM & 1 & & & & & & & & & & \\
\hline NP & 0,7855 & 1 & & & & & & & & & \\
\hline VA & $-0,1954$ & $-0,0466$ & 1 & & & & & & & & \\
\hline CC & $-0,2743$ & $-0,2872$ & 0,6475 & 1 & & & & & & & \\
\hline DP & $-0,1756$ & $-0,1146$ & 0,5288 & 0,0748 & 1 & & & & & & \\
\hline GL & 0,4356 & 0,5352 & $-0,4400$ & $-0,2088$ & $-0,4064$ & 1 & & & & & \\
\hline EP & 0,4614 & 0,4739 & 0,3025 & 0,2208 & $-0,0388$ & 0,4403 & 1 & & & & \\
\hline PM & 0,3207 & 0,3770 & 0,5598 & 0,4241 & 0,0417 & $-0,0053$ & 0,5066 & 1 & & & \\
\hline SC & 0,4926 & 0,4882 & $-0,2815$ & $-0,0692$ & $-0,5504$ & 0,8578 & 0,5817 & 0,2573 & 1 & & \\
\hline EM & 0,6581 & 0,5859 & 0,1724 & 0,2280 & 0,0761 & 0,3214 & 0,5008 & 0,5766 & 0,4360 & 1 & \\
\hline ARC & 0,6931 & 0,6250 & $-0,2224$ & 0,0991 & $-0,5145$ & 0,7096 & 0,4957 & 0,3184 & 0,7496 & 0,7013 & 1 \\
\hline
\end{tabular}

Fuente. Elaboración propia de los autores.

Se deduce que el criterio de los empresarios se acerca levemente a la teoría, cuando se logra establecer una correlación directa entre la investigación de mercados y el desarrollo de nuevos productos; sin embargo, las respuestas muestran la mayor relación entre el factor GL y el SC, situación que podría explicarse desde el punto de vista de la realidad empresarial y más, con el enfoque de seguimiento pos venta cuando se pretende satisfacer a plenitud y en el menor tiempo posible al cliente.

Escenario 2: en la tabla 5, se relacionan las variables seleccionadas como las competitivas y ajustadas, acorde a las tendencias del sector a nivel nacional e internacional, para entender si existe cohesión directa según el criterio de los empresarios. 
Tabla 5. Coeficiente de correlación de Pearson de factores de mercadeo incidentes en la competitividad, escenario 2

\begin{tabular}{|l|l|l|l|l|}
\hline & \multicolumn{1}{c|}{ VA } & \multicolumn{1}{c|}{ CC } & \multicolumn{1}{c|}{ GL } & ARC \\
\hline VA & 1 & & & \\
\hline CC & 0,64749621 & 1 & & \\
\hline GL & $-0,44002574$ & $-0,20877392$ & 1 & \\
\hline ARC & $-0,22243591$ & 0,09910389 & 0,70955582 & 1 \\
\hline
\end{tabular}

Fuente. Elaboración propia del autor.
Se deduce que los niveles de relación son positivos, pero muy leves, situación que puede llevar a que no haya dependencia alguna entre las variables. Para la gestión de mercadeo realizada por las empresas, desde el análisis estadístico, no existe una cohesión sistémica entre las variables, lo que lleva a que las acciones puedan actuar por separado, sin depender estrictamente las unas de las otras.

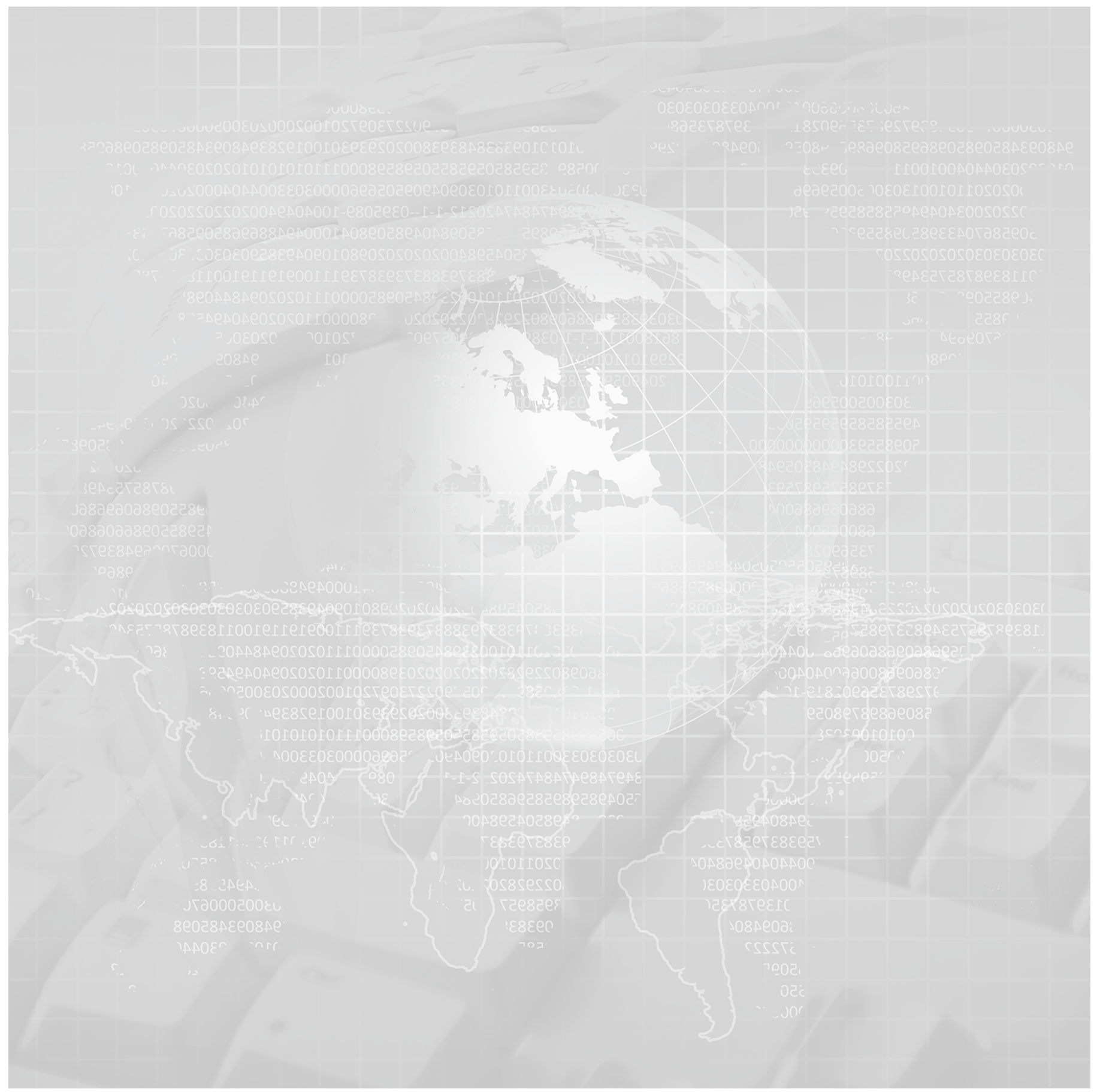




\section{CONCLUSIONES}

$\mathbf{L}$ a información del mercadeo sobre el sector, resalta la existencia de tendencias mundiales a las cuales la industria gráfica colombiana no ha logrado adaptarse lo suficiente para competir. Ellas son: impresión por demanda, digitalización de contenidos, conciencia ambiental y los servicios adicionales de impresión. Colombia, posee sus propias tendencias, no lejanas de lo que internacionalmente se mueve en el mercado pero de interés para el mercado nacional, como son: el transpromo, el libro digital, los Fotobooks y las aplicaciones de seguridad. Se requiere para la industria nacional, buscar la manera de adaptar servicios logísticos que son hoy por hoy, ampliamente utilizados a nivel global, pero escasamente usados en Colombia, suceso que generaría nuevas oportunidades de negocios para las empresas del sector.

Al analizar el sector gráfico en la ciudad de Barranquilla, la escala de medición arrojó a los siguientes factores de la gestión de mercadeo, como los incidentes en la competitividad:

- El aporte de valor agregado en los productos actuales con el $58 \%$.

- Los precios agresivos que pueda arriesgar ganancias con el $69 \%$.

- La gestión efectiva en la logística comercial, enfocada a reducir tiempos de entrega y con precios competitivos con el $62 \%$.
- La creatividad en la comercialización con el $82 \%$.

- El seguimiento a clientes en pre y pos venta, con el $63 \%$.

- El uso de herramientas informáticas para una mejor administración de los clientes con el 56\%.

Al comparar los factores anteriormente mencionados con la información sobre tendencias de mercado para la competitividad del sector en Colombia y a nivel internacional, se descartan dos de ellos (precios agresivos que pueda arriesgar ganancias y seguimiento a clientes en pre y pos venta); quedando así el resto de factores como los incidentes en la competitividad de las empresas del sector.

Finalmente, se realizan dos análisis correlaciónales para contrarrestar los criterios de los empresarios con enunciados de la teoría (escenario 1) y la sinergia entre los factores seleccionados por los empresarios, teniendo en cuenta la importancia del pensamiento sistémico en el diseño y ejecución de estrategias (escenario 2). Los resultados para el escenario 1, permitieron encontrar correlación entre las investigaciones de mercado y el desarrollo de nuevos productos, lo que iguala la teoría con lo que piensan los empresarios. Para el escenario 2 , se establece que aunque hay relaciones directas de dependencias entre las variables, son débiles, situación que lleva a que la gestión realizada no necesariamente necesita hacer sinergia de los factores seleccionados y de cierta manera, cualquiera de ellos por separado puede hacer a la empresa competitiva. 


\section{REFERENCIAS}

Arango, M., Branch, J., y Perez, G., (2008). Factores de innovación en marketing estratégico - un caso aplicado al sector de muebles hogar en Itagüi (Antioquia). Dyna. No 155 pp $29-36$

Cheraghi, H., Abdolhosseini, A., y Naderi, A., (2012). Gaining Competitive Advantage through Marketing Strategies in Container Terminal: A Case Study on Shahid Rajaee Port in Iran. International Business Research. Vol 5 No. 2 pp 179-191

Barrios, C., y López, B. (2007). Diseño de un modelo base de estructura organizacional para PYMES exportadoras del sector de confecciones de Barranquilla, con enfoque asociativo. Barranquilla.

Gómez, O. (2010). La productividad y competitividad de la industria de confecciones infantiles de Bucaramanga, en la dinámica de la gestión estratégica. Bucaramanga.

Iriarte, W., y Olave, J. (2001). Análisis de la gestión exportadora de las empresas del sector confección de Barranquilla vinculadas al programa EXPOPYME de la Universidad del Norte. 6.

Kotler, P. (2006). Dirección de Marketing. Pearson.

Metzger, M., y Donaire, V. (2007). Gerencia Estratégica de Mercadeo. CENGAGE Learning.

Ministerio de Comercio, C. (2009). Desarrollando sectores de clase mundial en Colombia, Informe final sector Textil, Confección, Diseño y Moda.

Olave, J. (2004). Propuesta de un modelo asociativo de gestión exportadora a partir del análisis del sector de confecciones del departamento del Atlántico (Colombia). Barranquilla.

Porter, M. (2010). Ventaja competitiva: Creación y sostenibilidad de un rendimiento Superior. Piramide.

Rivera, J. (2007). Dirección de marketing: Fundamentos y aplicaciones. ESIC.

Romero, L. E. (2006). Competitividad y productividad en empresas Familiares PyMEs. Revista EAN, 131-141.

Thompson, y Strickland. (2001). Administración estratégica. Conceptos y casos. Mexico: Mc Graw Hil.

Trout, A. R. (1998). Posicionamiento. Mc Graw Hill.

Villegas Londoño, D. I., y Toro Jaramillo, I. D. (2010). Las Pymes: una mirada a partir de la experiencia academica del $M B A$. Revista MBA EAFIT , 86-101.

Zapata, E. (2001). La efectividad del mercadeo en las pequeñas y medianas empresas (PyMEs) de los sectores industriales y de servicios de Boyacá, Colombia. Boyacá. 